\title{
Anterior Chamber Angle Assessment System
}

\author{
Huazhu $\mathrm{Fu}^{1}$, Yanwu $\mathrm{Xu}^{1}$, Damon Wing Kee Wong ${ }^{1}$, Jiang Liu ${ }^{2}$, Mani \\ Baskaran $^{3}$, Shamira A. Perera ${ }^{3}$, and Tin Aung ${ }^{3}$ \\ ${ }^{1}$ Institute for Infocomm Research, A*STAR, Singapore. \\ ${ }^{2}$ Cixi Institute of Biomedical Engineering, Ningbo Institute of Materials Technology \\ and Engineering, Chinese Academy of Sciences, China. \\ ${ }^{3}$ Singapore Eye Research Institute and Singapore National Eye Centre, Singapore.
}

\begin{abstract}
In this paper, we propose an automatic anterior chamber angle assessment system for Anterior Segment Optical Coherence Tomography (AS-OCT). In our system, the automatic segmentation method is used to segment the clinical structures, which are then used to recover standard clinical ACA measurements. Our measurements can not only support clinical assessments, but also be utilized as features for detecting anterior angle closure in automatic glaucoma diagnosis.
\end{abstract}

\section{Introduction}

Glaucoma is the second leading cause of blindness worldwide (behind cataracts) as well as the foremost cause of irreversible blindness. Since vision loss from glaucoma cannot be reversed, improved screening and detection methods for glaucoma are essential to preserve vision and life quality. Imaging and assessment of the ACA is thus critical for diagnosis of angle-closure glaucoma, which is one main subtype of primary glaucoma. We propose a system for automatic anterior chamber angle segmentation and measurement. In this paper, we propose a full automatic segmentation method to segment the clinical structures which are used to recover standard clinical ACA measurements. The key contributions of our system are to provide a full automatic segmentation and report the clinical ACA measurements, which can not only support clinicians in making anatomical assessments, but also be utilized as features for detecting anterior angle closure in automatic glaucoma diagnosis.

\section{Method}

Our system includes three main parts: ACA segmentation, clinical measurement, and glaucoma assessment, as shown in Fig. 1. For segmentation, we employ the automatic ACA structure segmentation method in [1], which is based on label transfer technique. The key idea is to make use of a reference image set containing manually labeled markers on the iris and corneal boundaries, through transfer of markers from the reference images most similar to the input. The transferred marker locations are refined based on structural information in the AS-OCT

X. Chen, M. K. Garvin, J. Liu, E. Trucco, Y. Xu (Eds.): OMIA 2016, Held in Conjunction with MICCAI 2016, Athens, Greece, Iowa Research Online, pp. 152-153, 2016. Available from: http://ir.uiowa.edu/omia/2016_Proceedings/2016/ 


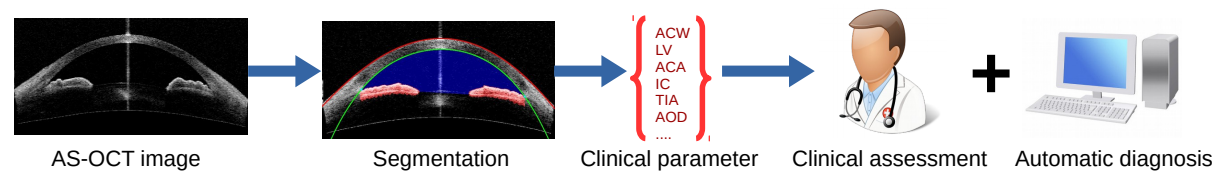

Fig. 1. Flowchart of our proposed method.

image, and then used to guide the segmentation of major ACA structures: the corneal boundary, iris region, and trabecular-iris contact. More details of the segmentation can be found in [1]. After ACA structure segmentation, several clinical ACA measurements are calculated, which are utilized for clinical assessment and have been shown to be discriminative features for automatic glaucoma classification. Finally, the clinical measurements for each ACA are obtained to clinician for making anatomical assessments, and also utilized as the clinical feature in a Linear SVM classifier for the angle-closure glaucoma classification.

\section{Evaluation}

In our evaluation, a total of 4137 AS-OCT images from 2113 subjects are collected from a Carl Zeiss Visante. Each AS-OCT image is split into two ACA sub-images (8274 ACA sub-images in total), since it contains two ACA regions and the right ACA sub-image is flipped horizontally. For each ACA sub-image, it is labeled as open-angle or angle-closure by a majority of three ophthalmologists. The dataset is divided equally and randomly into training and testing sets. We repeat the algorithm ten times and report the mean and standard deviations. The AUC and Accuracy scores of our system are $0.864 \pm 0.004$ and $0.856 \pm 0.007$. Our classification results are comparable to those of $\mathrm{Xu}$ et al. [3], which performs at 0.835 AUC score using HOG features in ACA images but cannot provide ACA measurements for clinical assessment. Our results are also comparable to those of Niwas et al. [2], which achieves 0.866 Accuracy by using a Laplacian score feature selection method. Note that the ACA measurements used in [2] are measured manually, so it is not an automatic method. The experiments on this clinical dataset demonstrates the effectiveness of our system.

\section{References}

1. Fu, H., Xu, Y., Wing, D., Liu, J., Baskaran, M., Perera, S., Aung, T.: Automatic anterior chamber angle structure segmentation in as-oct image based on label transfer. In: EMBC (2016)

2. Niwas, S., Lin, W., Kwoh, C., Kuo, J., Aquino, M., Sng, C., Chew, P.: Crossexamination for angle-closure glaucoma feature detection. IEEE J. Biomed. Health Inform. 20(1), 343-354 (2016)

3. Xu, Y., Liu, J., Tan, N., Lee, B., Wong, D., Baskaran, M., Perera, S., Aung, T.: Anterior chamber angle classification using multiscale histograms of oriented gradients for glaucoma subtype identification. In: EMBC. pp. 3167-3170 (2012) 